\title{
CARACTERIZAÇÃO DOS ÓBITOS MATERNOS EM TRÊS REGIÕES DE SAÚDE DO CENTRO-NORTE BAIANO
}

\author{
Vanessa Correia Coelho ${ }^{1}$, Magna Santos Andrade ${ }^{2}$, Chalana Duarte de Sena ${ }^{3}$, Laura Emanuela Lima Costa ${ }^{4}$, \\ Isaiane Santos Bittencourt ${ }^{3}$
}

\begin{abstract}
RESUMO: Objetivou-se descrever o perfil epidemiológico da mortalidade materna nos municípios que fazem parte da $15^{\mathrm{a}}, 16^{\mathrm{a}}$ e $28^{\mathrm{a}}$ Diretoria Regional de Saúde - Bahia, ocorridos entre os anos de 2006 e 2012. Trata-se de um estudo transversal, com dados secundários dos 113 óbitos maternos registrados no Sistema de Informação sobre Mortalidade, realizado de julho a setembro de 2013. Prevaleceram os óbitos em mulheres: entre 20-29 anos; raça negra; 4 a 7 anos de estudo; sem companheiro; tiveram entre 1 e 4 gestações; realizaram 4 a 6 consultas prénatal, iniciaram pré-natal até o $1^{\circ}$ trimestre; ocorrendo principalmente no puerpério (até 42 dias). Os resultados mostram que a mortalidade materna não é apenas um problema de saúde pública, mas uma questão social. O conhecimento deste estudo pode contribuir para que gestores, trabalhadores de saúde e sociedade estruturem conjuntamente políticas públicas e implementem estratégias para reduzir o número de óbitos maternos na região. DESCRITORES: Mortalidade materna; Sistemas de informação; Saúde da mulher; Saúde pública.
\end{abstract}

\section{CHARACTERIZATION OF MATERNAL DEATHS IN THREE HEALTHCARE REGIONS OF THE CENTER-NORTH OF BAHIA}

ABSTRACT: The present study aimed to describe the epidemiological profile of maternal mortality in the cities that constitute the 15th, 16th and 28th Regional Health Boards - Bahia, occurred between 2006 and 2012.Cross-sectional study with secondary data from 113 maternal deaths recorded in the Mortality Information System, conducted from July to September 2013. Deaths were more prevalent among black women aged 20-29 years old, with 4 to 7 years of education, living without a partner, who had 1 to 4 pregnancies; 4 to 6 prenatal appointments, started prenatal care up to the 1st trimester of the pregnancy. The deaths occurred mainly in the postpartum period (up to 42 days). The results showed that maternal mortality is not only a public health problem: it is also a social issue. The knowledge provided by this study may help managers, health workers and the society to develop joint public policies and implement strategies aimed to reduce the number of maternal deaths in the region.

DESCRIPTORS: Maternal mortality; Information systems; Women's health; Public health.

\section{CARACTERIZACIÓN DE LOS ÓBITOS MATERNOS EN TRES REGIONES DE SALUD DE CENTRO-NORTE DE BAHIA}

RESUMEN: Fue objetivo del estudio describirel perfil epidemiológico de la mortalidad materna en municipios que integran la $15^{\mathrm{a}}, 16^{\mathrm{a}}$ y $28^{a}$ "Junta Directiva Regional de Salud"- Bahia, entre los años de 2006 y 2012. Es un estudio transversal, con datos secundarios de 113 óbitos maternos registrados en el Sistema de Información sobre Mortalidad, realizado de julio a septiembre de 2013. Prevalecieronlos óbitos en mujeres: entre 20 y 29 años; de raza negra; 4 a 7 años de estudio; sin compañero; que tuvieron entre 1 y 4 gestaciones; realizaron 4 a 6 consultas de prenatal, empezaronprenatal hastael $1^{\circ}$ trimestre; que ocurrieron principalmente en el puerperio (hasta 42 días). Los resultados muestran que la mortalidad materna no essolamente un problema de salud pública, sino una cuestión social. El conocimiento de este estudio puede contribuir para que gestores, trabajadores de saludy sociedad estructuren conjuntamente políticas públicas así como implementen estrategias para reducir el número de óbitos maternos en la región.

DESCRIPTORES: Mortalidad materna; Sistemas de información; Salud de la mujer; Salud pública.

${ }^{1}$ Enfermeira. Universidade do Estado da Bahia. Senhor do Bonfim, BA, Brasil.

${ }^{2}$ Enfermeira. Mestre em Saúde Coletiva. Docente de Enfermagem da Universidade do Estado da Bahia. Senhor do Bonfim, BA, Brasil.

${ }^{3}$ Enfermeira. Mestre em Enfermagem. Docente de Enfermagem da Universidade do Estado da Bahia. Senhor do Bonfim, BA, Brasil.

${ }^{4}$ Enfermeira. Mestre em Enfermagem. Docente de Educação Física da Universidade do Estado da Bahia. Senhor do Bonfim, BA, Brasil. 


\section{- INTRODUÇÃO}

A mortalidade materna consiste em um grave problema de saúde pública, tendo indicadores elevados em diversos países. No ano de 2011, foram registrados aproximadamente 273.000 óbitos maternos em todo o mundo ${ }^{(1)}$.

A morte materna é definida como o óbito de uma mulher durante a gestação, parto ou puerpério (até 42 dias após o parto), independente da localização ou duração da gravidez, devido a complicações prévias ou desenvolvidas durante a gestação, excetuando acidentes ou incidentes. É considerada morte materna tardia quando o óbito ocorre entre 43 dias e menos de 1 ano após o parto, seja por causas obstétricas diretas ou indiretas ${ }^{(2)}$.

No Brasil os números são elevados. Em 2011 a Razão de Morte Materna (RMM) foi de 63,9 mortes por 100.000 nascidos vivos ${ }^{(3)}$ e no estado da Bahia, nesse mesmo ano, esse indicador alcançou 87,9 óbitos por 100.000 nascidos-vivos ${ }^{(4)}$, dados preocupantes ao considerar que a mortalidade materna é definida como baixa quando a RMM atinge valores até 20 óbitos por 100 mil nascidos vivos e mortalidade materna elevada ocorre em situações que ultrapassam 50 mortes por 100.000 nascidos vivos ${ }^{(5)}$.

A notificação e principalmente a investigação dos casos de óbitos maternos em tempo oportuno e preenchimento das informações de maneira correta são instrumentos fundamentais para o monitoramento da mortalidade materna, bem como para a quantificação real desses óbitos, perfil das mulheres acometidas e assistência prestada, informações fundamentais para a formulação e implementação de medidas para evitar o acontecimento de novos $\operatorname{casos}^{(5-7)}$.

Ao compreender as mortes maternas dentro do contexto das desigualdades socioeconômicas e demográficas, alguns fatores de risco podem ser destacados, tais como: idade avançada da mulher, baixa escolaridade e raça/cor negra ${ }^{(8,9)}$. Características obstétricas como a não realização do PréNatal (PN) ou número reduzido de consultas e a realização de cesariana também estão associados à mortalidade materna ${ }^{(8)}$.

O conhecimento dos aspectos relacionados aos óbitos maternos a partir da caracterização dos fatores sociodemográficos, gineco-obstétricos e descrição do momento do óbito podem viabilizar a identificação dos grupos populacionais mais vulneráveis à morte materna, sendo este entendimento imprescindível para a estruturação de políticas públicas e implementação de intervenções que objetivem a redução do número de casos.

Para a caracterização deste problema de saúde pública, propõe-se como questão norteadora para o desenvolvimento do estudo: Qual o perfil epidemiológico da mortalidade materna nos municípios que fazem parte da 15a $16^{\mathrm{a}}$ e $28^{\mathrm{a}}$ Diretoria Regional de Saúde (DIRES)-BA, ocorridos entre os anos de 2006 e 2012 ?

Neste contexto o objetivo deste estudo foi descrever o perfil epidemiológico da mortalidade materna nos municípios que fazem parte da $15^{\mathrm{a}}, 16^{\mathrm{a}}$ e $28^{\mathrm{a}}$ DIRES-BA, ocorridos entre os anos de $2006 \mathrm{e}$ 2012.

\section{○ MÉTODO}

Trata-se de um estudo descritivo, transversal, com a utilização de dados secundários, sendo incluídos todos os 113 óbitos maternos registrados no Sistema de Informação sobre Mortalidade (SIM), por meio das Declarações de Óbito (DO) e Fichas-síntese de Investigação (FI), ocorridos entre os anos de 2006 e 2012 nos municípios pertencentes às 15 ${ }^{\mathrm{a}}, 16^{\mathrm{a}}$ e $28^{\mathrm{a}}$ DIRES do estado da Bahia. O ano de 2006 foi escolhido por se tratar do período inicial das investigações de morte materna, e o ano de 2012 por ser o período mais recente com dados completos.

Os locais de estudo foram a $15^{a}, 16^{a}$ e $28^{a}$ DIRES, localizadas nas cidades de Juazeiro, Jacobina e Senhor do Bonfim, respectivamente, abrangendo 37 municípios baianos, situados na mesorregião Centro-Norte do estado. Destes, 9 pertencem à $15^{\circ}$ DIRES (Campo Alegre de Lourdes, Casa Nova, Curaçá, Juazeiro, Pilão Arcado, Remanso, Sento Sé, Sobradinho e Uauá), 19 à $16^{\circ}$ (Caém, Caldeirão 
Grande, Capim Grosso, Jacobina, Mairi, Miguel Calmon, Mirangaba, Morro Do Chapéu, Ourolândia, Piritiba, Quixabeira, São José do Jacuípe, Saúde, Serrolândia, Tapiramutá, Umburanas, Várzea Da Roça, Várzea Do Poço, Várzea Nova) e 9 formam a $28^{\circ}$ (Andorinha, Antônio Gonçalves, Campo Formoso, Filadélfia, Itiúba, Jaguarari, Pindobaçú, Ponto Novo e Senhor do Bonfim), sendo que juntos, estes municípios somam 1.152.382 habitantes ${ }^{(10-12)}$.

Como critérios de inclusão foram considerados: óbitos ocorridos em mulheres em qualquer faixa etária; no período da gestação ou no momento do parto; relacionados ao aborto; ocorridos durante o puerpério até 42 dias ou até menos de 1 ano após o parto (morte materna tardia) ${ }^{(2)}$. Os critérios de exclusão foram: mulheres que não foram a óbito no período gravídico-puerperal; óbitos ocorridos durante a gestação, parto ou pós-parto por causas acidentais ou incidentais; óbitos maternos que não ocorreram nas regiões das $15^{a}, 16^{a}$ e $28^{a}$ DIRES do estado da Bahia.

Para descrever o perfil epidemiológico das mortes maternas foram estudadas as seguintes variáveis: sociodemográficas (idade, raça/cor, anos de estudo e situação conjugal); gineco-obstétricas (número de gestações anteriores, número de consultas PN, início do PN, tipo de parto e momento do óbito); características dos óbitos (recebeu assistência médica durante a doença que ocasionou a morte, local de ocorrência do óbito, causa da morte e causas diretas da morte materna).

A causa básica de morte foi classificada como obstétrica direta (decorrente de complicações obstétricas durante a gravidez, parto e puerpério) e obstétrica indireta (decorrente de doenças pregressas à gestação, ou que se desenvolveram durante a gestação devido às alterações fisiológicas da gravidez) $)^{(2)}$.

Os dados foram coletados a partir das DO e das FI entre os meses de julho e setembro de 2013, armazenados em banco de dados, analisados e processados no programa Statical Package for Social Sciences (SPSS) versão 9.0.

Em seguida, foram feitas as correções dos erros de digitação e desenvolvida análise exploratória e descritiva da amostra em estudo, verificando-se as frequências simples das variáveis estudadas.

O projeto foi aprovado (parecer $n^{\circ}$ 519.522) pelo Comitê de Ética em pesquisa da Universidade do Estado da Bahia em 03 de Fevereiro de 2014 e respeitou o que estabelece a Resolução 466/2012 do Conselho Nacional de Saúde/Ministério da Saúde (MS), que determina as diretrizes e normas das pesquisas envolvendo seres humanos ${ }^{(13)}$.

\section{RESULTADOS}

Entre os anos de 2006 e 2012 foram registrados no SIM 113 óbitos maternos nos 37 municípios que constituem as três DIRES estudadas, sendo que destes casos 28 (24,8\%) foram investigados.

A análise por DIRES aponta que a baixa investigação foi um problema evidenciado nos três campos do estudo, pois 14 (30,4\%) óbitos maternos ocorridos na $15^{\mathrm{a}}$ DIRES foram investigados, 5 (15,6\%) na $16^{\mathrm{a}}$ e $9(25,7 \%)$ na $28^{\text {a }}$ (Figura 1$)$.

Observou-se a ausência de registro de diversas variáveis nas DO pesquisadas, mas em algumas foi significativa a inexistência de informação que impediu sua utilização para a caracterização do perfil dos óbitos maternos. Dentre as variáveis com preenchimento em branco, destacam-se: 40 (35\%) ocupação habitual/ramo de atividade e $54(47,7 \%)$ bairro/distrito.

Dentre os casos de morte materna 49 (43,4\%) ocorreram em mulheres entre 20-29 anos, 85 (75,2\%) eram negras, 24 (21,2\%) tinham de 4 a 7 anos de estudo e 55 (48,7\%) não possuíam companheiro (Tabela 1).

Verificou-se através das FI que entre os óbitos maternos predominou: 19 (67,9\%) mulheres com 1 a 4 gestações; 13 (46,4\%) realizaram de 4 a 6 consultas de PN; $13(61,9 \%)$; iniciaram o PN no primeiro trimestre de gestação; 51 (45,1\%) morreram nos primeiros 42 dias de pós-parto; 50 (44,2\%) óbitos ocorreram na gestação, parto ou aborto; 12 (52,2\%) dentre aquelas cujo óbito foi no momento do parto foram cesarianas (Tabela 2). 


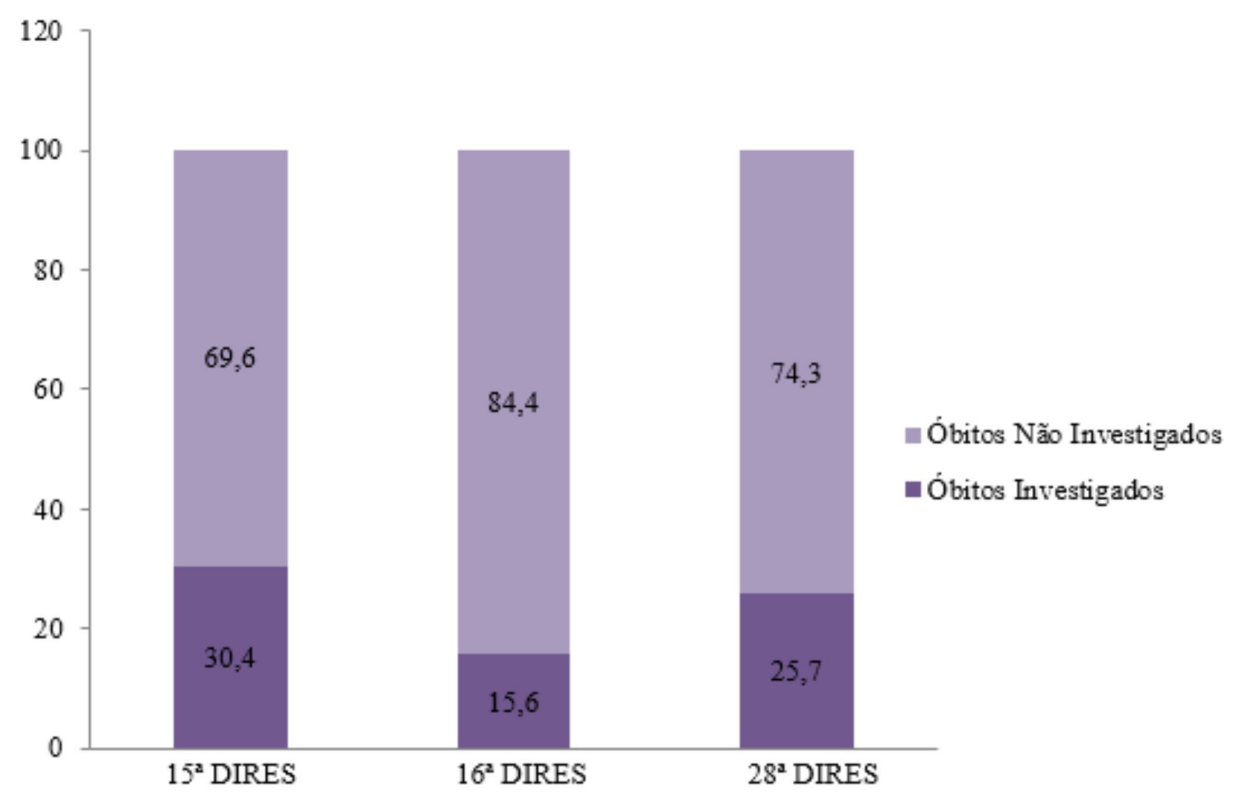

Fonte: Sistema de Informação de Mortalidade/Ministério da Saúde 2013.

Figura 1 - Percentual de óbitos maternos investigados nos municípios da 15ª $16^{\mathrm{a}}$ e $28^{\mathrm{a}}$ DIRES. Bahia, Brasil, 2006 a 2012. Fonte: Sistema de Informação sobre Mortalidade/Ministério da Saúde - 2013

Tabela 1 - Caracterização sociodemográfica dos óbitos maternos nos municípios da $15^{\mathrm{a}}, 16^{\mathrm{a}}$ e $28^{\mathrm{a}}$ DIRES. Bahia, Brasil, 2006 a 2012

\begin{tabular}{lcc}
\hline Variável & $\mathbf{n}$ & $\mathbf{\%}$ \\
\hline Faixa etária (113) & & \\
\hline 10-19 anos & 14 & 12,4 \\
\hline 20-29 anos & 49 & 43,4 \\
\hline 30-39 anos & 36 & 31,8 \\
\hline 40 anos ou mais & 14 & 12,4 \\
\hline Raça/cor (113) & & \\
\hline Branca & 19 & 16,8 \\
\hline Negra & 85 & 75,2 \\
\hline Sem resposta & 9 & 8 \\
\hline Anos de estudo (113) & & \\
\hline Nenhum & 8 & 7,1 \\
\hline 1 a 3 anos & 22 & 19,5 \\
\hline 4 a 7 anos & 24 & 21,2 \\
\hline 8 a 11 anos & 14 & 12,4 \\
\hline 12 anos ou mais & 13 & 11,5 \\
\hline Sem resposta/lgnorado & 32 & 28,3 \\
\hline Situação conjugal (113) & & \\
\hline Sem companheiro & 55 & 48,7 \\
\hline Com companheiro & 35,4 \\
\hline Sem resposta/lgnorado & 15,9 \\
\hline
\end{tabular}

Fonte: Sistema de Informação sobre Mortalidade/Ministério da Saúde - 2013.
Observou-se que em $84(74,3 \%)$ casos, as mulheres receberam assistência médica durante a doença que levou à morte, $98(86,7 \%)$ óbitos ocorreram no hospital, prevalecendo como causa morte a obstétrica direta com $71(62,8 \%)$ casos e dentre as causas diretas, a mais prevalente foi eclâmpsia/ pré-eclâmpsia, sendo que 24 (33,8\%) óbitos ocorreram por essas complicações (Tabela $3)$. 
Tabela 2 - Caracterização gineco-obstétrica dos óbitos maternos nos municípios da 15 ${ }^{\mathrm{a}}, 16^{\mathrm{a}}$ e $28^{\mathrm{a}}$ DIRES.Bahia, Brasil, 2006 a 2012

\begin{tabular}{|c|c|c|}
\hline Variáveis & $\mathbf{n}$ & $\%$ \\
\hline \multicolumn{3}{|l|}{ Número de gestações anteriores (28)* } \\
\hline Primigesta & 5 & 17,8 \\
\hline De 1 a 4 gestações & 19 & 67,9 \\
\hline 5 ou mais & 4 & 14,3 \\
\hline \multicolumn{3}{|l|}{ Número de consultas pré-natal (28)* } \\
\hline Nenhuma & 7 & 25 \\
\hline De 1 a 3 & 2 & 7,2 \\
\hline De 4 a 6 & 13 & 46,4 \\
\hline 7 e mais & 6 & 21,4 \\
\hline \multicolumn{3}{|c|}{ Início do pré-natal (mês de gestação) (21)** } \\
\hline Até o $3^{\circ}$ mês & 13 & 61,9 \\
\hline $4^{\circ}$ mês ou mais & 8 & 38,1 \\
\hline \multicolumn{3}{|l|}{ Momento do óbito (113)*** } \\
\hline Gestação/parto/aborto & 50 & 44,2 \\
\hline No puerpério, até 42 dias & 51 & 45,1 \\
\hline No puerpério, de 43 dias a 1 ano & 5 & 4,5 \\
\hline Sem informação & 7 & 6,2 \\
\hline \multicolumn{3}{|l|}{ Tipo de parto $(23)^{* *}$} \\
\hline Vaginal & 11 & 47,8 \\
\hline Cesárea & 12 & 52,2 \\
\hline
\end{tabular}

Fonte: Sistema de Informação sobre Mortalidade/Ministério da Saúde - 2013.

*Total de óbitos investigados $=28$.

**Número de Fl em que a informação estava disponível.

***Fonte: Dados obtidos através das DO registradas no SIM.
Tabela 3 - Caracterização dos óbitos maternos ocorridos nos municípios da 15 ${ }^{\mathrm{a}}, 16^{\mathrm{a}}$ e $28^{\mathrm{a}}$ DIRES. Bahia, Brasil, 2006 a 2012

\begin{tabular}{lcc} 
Variáveis & $\mathbf{n}$ & $\mathbf{\%}$ \\
\hline $\begin{array}{l}\text { Recebeu assistência médica durante a } \\
\text { doença que ocasionou a morte (113) }\end{array}$ & & \\
\hline Sim & 84 & 74,3 \\
\hline Não & 9 & 8 \\
\hline Sem resposta/Ignorado & 20 & 17,7 \\
\hline Local de ocorrência do óbito (113) & & \\
\hline Hospital & 98 & 86,7 \\
\hline Via pública/Domicílio & 11 & 9,7 \\
\hline Outros & 4 & 3,6 \\
\hline Causa da morte (113) & & \\
\hline Obstétrica direta & 71 & 62,8 \\
\hline Obstétrica indireta & 35 & 31 \\
\hline Morte inespecificada & 7 & 6,2 \\
\hline Causas diretas da morte materna (71) & & \\
\hline Eclampsia/Pré-eclâmpsia & 24 & 33,8 \\
\hline Hemorragias & 6 & 8,5 \\
\hline Aborto & 5 & 7,1 \\
\hline DHEG & 3 & 4,2 \\
\hline Infecção & 26 & 5,6 \\
\hline Inércia uterina & & 4,2 \\
\hline Outras causas & & \\
\hline
\end{tabular}

Fonte: Sistema de Informação sobre Mortalidade/Ministério da Saúde - 2013.

\section{DISCUSSÃO}

No presente estudo, o preenchimento inadequado das $\mathrm{DO}$ e o reduzido número de óbitos investigados $(28,4 \%)$ foram fatores que dificultaram o maior detalhamento do perfil epidemiológico das mortes maternas na região estudada.

No entanto, esta não é uma realidade apenas dos municípios localizados no Centro-Norte baiano, pois o elevado sub-registro (omissão do registro do óbito em cartório) e a subinformação (decorrente do preenchimento incorreto da DO) são alguns dos fatores que interferem de maneira negativa no conhecimento da real situação da mortalidade materna em todo o território nacional ${ }^{(2)}$.

A faixa etária que apresentou maior percentual de óbitos maternos foi entre 20-29 anos de idade $(43,4 \%)$ semelhante ao observado em todo o território nacional, que a maior frequência de óbitos também ocorre nessa mesma fase da vida $(41,8 \%)^{(7)}$.

As mortes maternas ocorridas entre os 20-29 anos podem ser atribuídas a falhas da assistência durante o ciclo gravídico-puerperal, pois esta não é considerada uma fase de risco gestacional para a mulher ${ }^{(5)}$, sendo apontada como idade de risco gestantes menores de 15 anos ou com mais de 35 $\operatorname{anos}^{(14)}$.

As mulheres negras dos municípios estudados somaram $75,2 \%$ dos óbitos maternos ocorridos, 
número 4 vezes superior às brancas. No estado do Mato Grosso, 63\% dos óbitos maternos foram em mulheres negras, contrapondo-se a $29,7 \%$ de mulheres brancas ${ }^{(15)}$.

A vulnerabilidade das mulheres negras ao óbito materno está intimamente ligada à condição social e modo de vida em que este grupo está inserido ${ }^{(15)}$. As baixas condições socioeconômicas, o pouco acesso à educação de qualidade, a reduzida inserção nos serviços de saúde e o conhecimento precário em relação à adoção de práticas contraceptivas são alguns aspectos que podem estar associados à maior exposição das mulheres negras à mortalidade materna.

Embora, a maioria das DO tenham apresentado número considerável de "sem resposta/ignorado" $(28,3 \%)$ em relação à variável escolaridade, observou-se que parte das mulheres possuía entre 4 a 7 anos de estudo (21,2\%), percentual semelhante ao encontrado em estudo da região Norte do Brasil (30\%), no estado do Pará $(34,5 \%)$ e em Recife-PE $(40,5 \%)^{(7,9,5)}$. O nível de escolaridade relaciona-se à morte materna, pois mulheres com pouco grau de escolaridade buscam menos os serviços de saúde e possuem acesso restrito às informações acerca da saúde sexual e reprodutiva ${ }^{(5,16)}$.

Mulheres com poucos anos de estudo têm menos conhecimento sobre práticas de saúde, dessa forma, a baixa escolaridade pode interferir na compreensão das informações que lhes são fornecidas pelos profissionais durante o PN e puerpério, consequentemente comprometendo o autocuidado e tornando-as mais vulneráveis à morbimortalidade, principalmente as negras, uma vez que o número de analfabetismo entre este grupo é duas vezes maior que entre as brancas ${ }^{(17)}$.

Mulheres sem companheiro representaram a maior parte dos óbitos neste estudo (48,7\%), assim como no Pará onde $66 \%$ das mortes ocorreram em mulheres solteiras ${ }^{(9)}$. A presença do companheiro configura-se como fator de proteção para a mulher durante o período gravídico e pós-parto, pois o parceiro na maioria das situações é alguém que cuida e busca os serviços de saúde quando as mulheres necessitam, atuando por consequência na redução de complicações e óbito. Assim, mulheres sem companheiro estão mais suscetíveis ao óbito materno ${ }^{(18)}$.

$\mathrm{Na}$ presente pesquisa, parte das mulheres tiveram de 1 a 4 gestações prévias $(67,9 \%)$, achado semelhante a pesquisa realizada em Recife-PE, onde $54,1 \%$ das mulheres que foram a óbito tinha o mesmo número de gestações anteriores ${ }^{(5)}$, não sendo observada a multiparidade, um conhecido fator de risco para a mortalidade materna, enquanto característica predominante entre as mulheres estudadas.

É preconizado que o número de consultas PN seja superior a $6^{(14)}$, porém, no presente estudo $46,4 \%$ das mulheres que foram a óbito realizaram de 4 a 6 consultas, número este superior ao encontrado em Recife-PE onde $31,5 \%$ das mulheres realizaram de 1 a 3 consultas de $\mathrm{PN}^{(5)}$.

Observou-se também, que as consultas de PN foram iniciadas precocemente (até o $3^{\circ}$ mês de gestação) (61,8\%), assemelhando-se aos achados de estudo em Recife-PE onde $67,6 \%$ das consultas foram iniciadas até o $4^{\circ}$ mês de gestação ${ }^{(5)}$.

O MS recomenda que as consultas sejam iniciadas preferencialmente no primeiro trimestre da gestação a fim de que as avaliações necessárias sejam realizadas, e este acompanhamento deve continuar até o $42^{\circ}$ dia após o parto, período de realização da consulta puerperal ${ }^{(14)}$.

No entanto, não apenas o quantitativo de consultas deve ser priorizado, mas também é necessário voltar a atenção para a qualidade de cada consulta realizada, desenvolvendo assistência humanizada, visando a melhores resultados para redução da morbimortalidade do binômio mãe e filho ${ }^{(7)}$.

A cesariana, assim como qualquer outra cirurgia abdominal, envolve riscos e requer cuidados, pois possui taxa de mortalidade materna e fetal maior que o parto vaginal(16). A depender do caráter de urgência e avaliando o risco-benefício, a cesariana pode ser indicada ${ }^{(5)}$, o que pode ser um dos fatores a justificar o quantitativo elevado de óbitos em $52,3 \%$ das mulheres que foram submetidas a este procedimento.

Assim como em pesquisa realizada em Juiz de Fora-MG $(69,1 \%)$ prevaleceram as mortes ocorridas durante o puerpério até 42 dias após o parto $(43,1 \%)^{(19)}$. O período puerperal é uma fase delicada em que os profissionais devem estar atentos. No âmbito da atenção básica, devem ser realizadas duas consultas 
de puerpério e cabe aos profissionais verificar e orientar as mulheres quanto ao aparecimento de sinais e sintomas que podem indicar complicações, tais como febre, dor ou infecção em episiotomia ou incisão de cesariana, sangramento vaginal intenso, entre outros ${ }^{(14)}$.

Neste contexto, devem ser desenvolvidos estudos que aprofundem a qualidade da assistência ao puerpério nos hospitais e unidades de saúde dos municípios que constituem o Centro-Norte baiano, objetivando a identificação de possíveis falhas na assistência para a estruturação de estratégias que melhorem a qualidade dos serviços oferecidos.

Dentre os casos estudados, $86,7 \%$ das mortes maternas pesquisadas ocorreram no hospital. No Pará, mais de $90 \%$ dos óbitos maternos estudados ocorreram em unidades hospitalares, podendose observar que as causas que levam essas mulheres ao óbito são de alta gravidade, necessitando deste modo de atenção especializada ${ }^{(9)}$, como foi visto de acordo com as principais causas de óbito levantadas.

Mesmo a maioria das mulheres recebendo assistência médica durante a doença que levou ao óbito (74,3\%), 62,8\% morreram por causas diretas dentre as quais prevaleceram os distúrbios hipertensivos (eclampsia e pré-eclâmpsia) $(33,8 \%)$, seguidos de hemorragias $(8,5 \%)$. Pesquisa realizada no Ceará evidenciou que $50 \%$ das mortes maternas ocorridas em 2011 foram por causas diretas ${ }^{(6)}$. No Brasil, as três principais causas de óbito materno em ordem decrescente são: a hipertensão gestacional, a hemorragia e a infecção puerperal(20).

As mortes maternas por causas diretas são em sua maioria evitáveis e possuem estreita relação com fatores sociais aos quais grupos específicos, como mulheres negras, pobres, indígenas, dentre outras, podem estar expostas: pouco acesso a serviços de saúde, inadequação do serviço prestado, reduzida sensibilização dos profissionais em relação às necessidades específicas dos diversos grupos, tudo isso repercutindo de maneira negativa na saúde materna ${ }^{(15,5)}$.

\section{- CONCLUSÃO}

O estudo mostrou que o perfil da mortalidade materna na região estudada refere-se a mulheres adultas jovens, negras, com baixa escolaridade, sem companheiro, que tiveram de 1 a 4 gestações, realizaram de 4 a 6 consultas de $\mathrm{PN}$ e iniciaram o PN precocemente. Os óbitos ocorreram principalmente no puerpério, a maioria das mulheres recebeu assistência médica para a patologia que levou à morte, a causa obstétrica direta foi a mais observada destacando-se a eclampsia/pré-eclâmpsia.

A partir dos dados encontrados, observa-se a necessidade de aprimorar o registro de informações acerca dos óbitos maternos, e ampliação da investigação dos casos, além de destacar a morte materna enquanto problema não apenas de saúde, mas também social, pois as principais vítimas foram mulheres negras, com baixa escolaridade e sem companheiro.

Pesquisas sobre a qualidade da assistência ao PN, parto e puerpério devem ser ampliadas, já que a maioria das mortes ocorreu por causas diretas e nem todas as mulheres tiveram assistência hospitalar no momento do óbito.

Os resultados deste estudo podem ser utilizados para o planejamento de ações que envolvam a promoção da saúde assim como embasamento para a avaliação de políticas públicas existentes e reestruturação de novas políticas de saúde destinadas a reduzir os elevados indicadores de óbitos maternos no Centro-Norte baiano.

\section{REFERÊNCIAS}

1. Lozano R, Wang H, Foreman KJ, Rajaratnam JK, Naghavi M, Marcus JR, et al. Progress towards Millennium Development Goals 4 and 5 on maternal and child mortality: an updated systematic analysis. The Lancet. 2011; 378(9797): 1139-65. 
2. Ministério da Saúde (BR). Secretaria de Atenção à Saúde. Departamento de Ações Programáticas Estratégicas. Manual dos comitês de mortalidade materna. $3^{a}$ ed. Brasília: Ministério da Saúde; 2009.

3. Escalante JC. Mortalidade materna no Brasil. Coordenação Geral de Informações e Análise Epidemiológica. Secretaria de Vigilância em Saúde. Ministério da Saúde; 2013.

4. Szwarcwald CL, Escalante JJC, Rabello Neto DL, Souza Junior PRB, Victora CG. Estimação da razão de mortalidade materna no Brasil, 2008-2011. Cad. Saúde Pública. [Internet] 2014; 30(Suppl1) [acesso em 15 nov 2015]. Disponível: http://dx.doi.org/10.1590/0102-311X00125313.

5. Correia RA, Araújo HC, Furtado BMA, Bonfim C. Características epidemiológicas dos óbitos maternos ocorridos em Recife, PE, Brasil (2000-2006). Rev. bras. enferm. 2011; 64(1):91-7.

6. Governo do Estado do Ceará. Secretaria da Saúde. Informe Epidemiológico Mortalidade Materna. Coordenadoria de Promoção e Proteção à Saúde/Núcleo de Vigilância Epidemiológica / SESA/Ce. 2013:1-22.

7. Ferraz L, Bordignon M. Mortalidade materna no Brasil: uma realidade que precisa melhorar. Rev. Baiana de Saúde Pública. 2012; 36(2): 527-38.

8. Leite RMB, Araújo TVB, Albuquerque RM, Andrade ARS, Duarte Neto PJ. Fatores de risco para morbidade materna em área urbana do Nordeste do Brasil. Cad. Saúde Pública. [Internet] 2011; 27(10) [acesso em 26 nov 2015]. Disponível: http://dx.doi.org/10.1590/S0102-311X2011001000011.

9. Botelho NM, Silva IFMM, Tavares JR, Lima LO. Morte materna no estado do Pará: aspectos epidemiológicos. Rev. para. med. 2013;27(1):1-11.

10. Ministério da Saúde (BR). Regiões de Saúde do Estado da Bahia. [Internet] 2013 [acesso em 23 abr 2013 ]. Disponível: http://www1.saude.ba.gov.br/mapa_bahia/Resultado.asp?DIRES=15+-+JUAZEIRO.

11. Ministério da Saúde (BR). Regiões de Saúde do Estado da Bahia. [Internet] 2013 [acesso em 10 out 2012]. Disponível: http://www1.saude.ba.gov.br/mapa_bahia/Resultado.asp?DIRES=16+-+JACOBINA.

12. Ministério da Saúde (BR). Regiões de Saúde do Estado da Bahia. [Internet] 2013 [acesso em 07 mai 2013 ]. Disponível: http://www1.saude.ba.gov.br/mapa_bahia/.

13. Ministério da Saúde (BR). Conselho Nacional de Saúde. Diretrizes e normas regulamentadoras de pesquisa envolvendo seres humanos. Resolução n. 466, de 12 de dezembro de 2012. Brasília; 2012.

14. Ministério da Saúde (BR). Secretaria de Atenção à Saúde. Departamento de Atenção Básica. Atenção ao prénatal de baixo risco. Brasília: Editora do Ministério da Saúde; 2012.

15. Texeira NZF, Pereira WR, Barbosa DA, Vianna LAC. Mortalidade materna e sua interface com a raça em Mato Grosso. Rev. Bras. Saúde Matern. Infant. 2012; 12(1): 27-35.

16. Ziegel EE, Cranley MS. Enfermagem Obstétrica. Tradução por J. Israel Lemos. [Reimpr.]. Rio de Janeiro: Guanabara Koogan; 2011.

17. Marinho ACN, Paes NA. Mortalidade materna no estado da Paraíba: associação entre variáveis. Rev. esc. enferm. USP. 2010; 44(3): 732-8.

18. Carreno I, Bonilha ALL, Costa JSD. Perfil epidemiológico das mortes maternas ocorridas no Rio Grande do Sul, Brasil: 2004-2007. Rev. bras. epidemiol. 2012; 15(2): 396-406.

19. Faria DR, Sousa RC, Costa TJNM, Leite ICG. Mortalidade materna em cidade-polo de assistência na região Sudeste: tendência temporal e determinantes sociais. Rev. Med. Minas Gerais. 2012; 22(1): 18-25.

20. World Health Organization (WHO). World Bank. UNICEF. United Nations Population Fund. Trends in maternal mortality: 1990 to 2010. Geneva: WHO; 2012. 